\title{
The reliability of single-field fundus photography in screening for diabetic retinopathy: the Central Australian Ocular Health Study
}

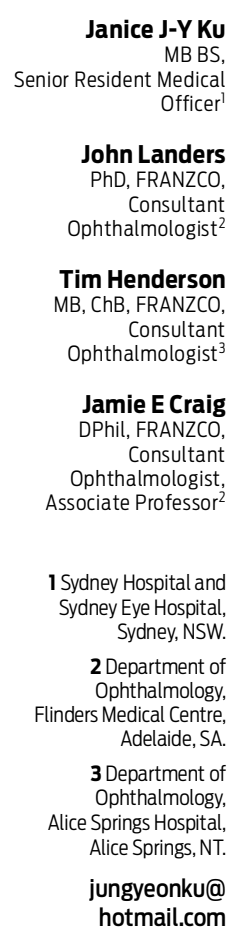

MJA 2013; 198: 93-96 doi: 10.5694/mjal2.10607

Editorial p 69 Research $p 97$

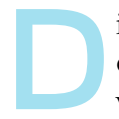

iabetic retinopathy (DR) is one of the leading causes of preventable vision loss in Australia. $^{1,2}$ The global prevalence of diabetes mellitus (DM) is on the rise, with 366 million $(4.4 \%$ of the estimated world population) expected to be affected by the year 2030. ${ }^{3}$ In Australia, a nationwide cross-sectional survey in 2002 showed that the prevalence of diabetes in Australia had more than doubled since 1981. At the time of the survey, $8.0 \%$ of adult men and $6.8 \%$ of adult women living in Australia had diabetes, and $15.3 \%$ of people with diabetes had DR. ${ }^{4}$

$\mathrm{DR}$ is detected at similar rates in both Indigenous and non-Indigenous Australians with DM. ${ }^{5,6}$ However, the burden of DR is much higher among the Indigenous Australian population because of the higher prevalence of DM within this group. A recent eye health survey in Indigenous Australians showed the prevalence of selfreported diabetes to be more than eight times higher (37\%) than in nonIndigenous Australians. This is a striking figure, as 30 years ago, only $0.03 \%$ of Indigenous people had diabetes. ${ }^{5}$

DR has little or no symptoms until vision loss develops, so regular DR screening is critical for early diagnosis and treatment. ${ }^{2,3}$ Vision loss can be prevented in up to $70 \%$ of people who are at risk through timely intervention. ${ }^{2,7}$ However, the rates of adherence to regular eye examinations in those with DR consistently fall below the recommended rate, and are as low as $50 \%$ in some studies. ${ }^{8,9}$ In particular, there is a significant shortfall in the delivery of DR screening to remote regions of Australia. This may have resulted from a shortage of service providers, obstacles in accessing centrally located specialist services and underutilisation of visiting services. Improv-

\begin{abstract}
Objective: To assess the accuracy of grading diabetic retinopathy (DR) using single-field digital fundus photography compared with clinical grading from a dilated slit-lamp fundus examination in Indigenous Australians living in Central Australia.

Design, setting and participants: Cross-sectional study comparing DR grades in participants with diabetes mellitus presenting for examination at remote community clinics from 1 July 2005 to 30 June 2008.

Main outcome measures: Sensitivity and specificity of grading using digital photography compared with the clinical gold standard of slit-lamp fundus examination.

Results: Of the 1884 participants recruited for the study, 1040 had self-reported diabetes mellitus and, of those, 360 had fundus photographs available (706 eyes) that were able to be graded. On clinical grading, 163 eyes had any DR and 51 eyes had vision-threatening DR (VTDR). The sensitivity and specificity for detecting any DR were 74\% (95\% Cl, 67\%-80\%) and 92\% (95\% Cl, 90\%$94 \%$ ), respectively. The sensitivity and specificity for detecting VTDR were $86 \%$ (95\% Cl, 77\%-96\%) and 95\% (95\% Cl, 93\%-97\%), respectively.

Conclusion: Single-field digital fundus photography is a valid screening tool for DR in remote communities of central Australia and may be used to provide eye care services to this region with acceptable accuracy.
\end{abstract}

ing the availability of fundus cameras and training local staff in their use may help overcome some of the barriers to DR screening.

An ideal screening method requires acceptable sensitivity and specificity, and cost-effectiveness. A recent meta-analysis confirmed the use of retinal photography as a valid screening tool for DR in resourcepoor settings. ${ }^{10}$ Our study was designed to evaluate the validity of single-field fundus photography as a screening tool for DR in remote Central Australian communities.

\section{Methods}

The design, recruitment process and baseline characteristics of the Central Australian Ocular Health Study (CAOHS) have previously been described in detail. ${ }^{11}$ The CAOHS took place in remote communities of Central Australia, excluding the relatively urbanised area of Alice Springs. The participants were recruited during once-weekly remote clinic visits over 36 months from 1 July 2005 to 30 June 2008. Ethics approval for the study was obtained from the Central Australian Human Research Ethics Committee according to the tenets of the Declaration of Helsinki. The aims of the study were explained to participants with the help of an interpreter when needed, and written informed consent was obtained.

All participants underwent detailed ocular examination. Baseline acuity was measured using a tumbling E acuity chart at 3 metres in a well lit room. An optometrist performed subjective refraction and determined refracted visual acuity. The optometrist performed a slit lamp examination of the anterior segment, followed by pupil examination using a hand torch. After an assessment of anterior chamber depth, the pupils were dilated using tropicamide $1.0 \%$ and phenylephrine $2.5 \%$ solution. The visiting ophthalmologist (TH) performed stereoscopic slit-lamp fundoscopy using a 90- 
1 Fundus photographs (examples from study cohort) showing a normal fundus (A), clinically significant macular oedema (B) and proliferative diabetic retinopathy (C)
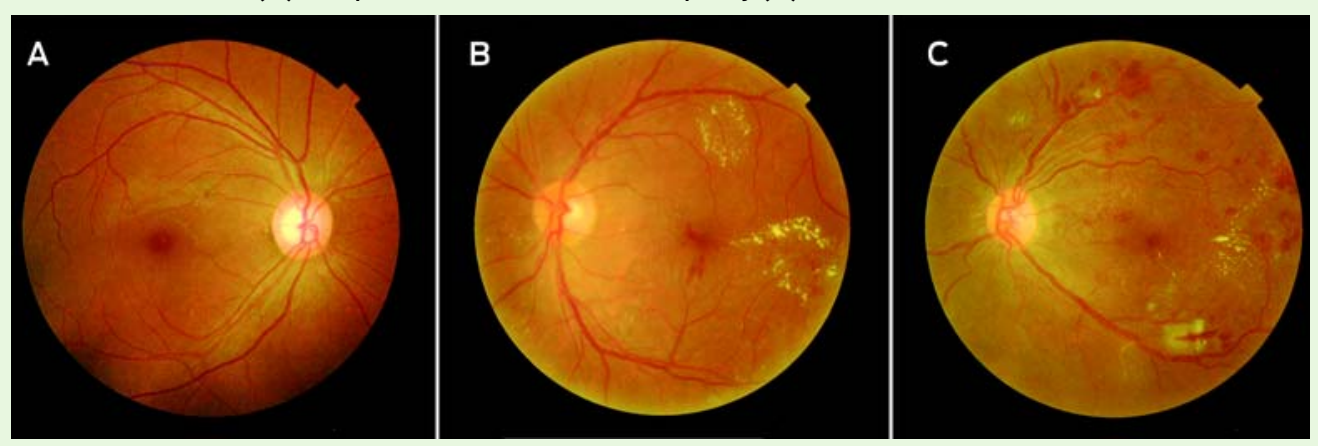

dioptre fundoscopy lens. The presence and degree of DR was graded using the Early Treatment of Diabetic Retinopathy Study (ETDRS) adaptation of the modified Airlie House classification of $\mathrm{DR}^{12}$ by clinical comparison with standardised photographs (Box 1). The DR was graded as either no DR (level 10-13), minimal non-proliferative DR (NPDR) (level 14-19), mild NPDR (level 20-39), moderate NPDR (level 40-49), severe NPDR (level 50-59) and proliferative DR (PDR) (level 60-85). Clinically significant macular oedema (CSMO) was defined as any retinal thickening within $500 \mu \mathrm{m}$ of the fovea associated with retinal thickening that is at least one disc area in size within one disc diameter of the fovea.

For the purposes of data analysis, the NPDR and PDR groups were collapsed into "any DR". CSMO and PDR groups were also collapsed into one category, named "vision-threatening DR" (VTDR).

Where possible, each participant with DM underwent single-field 45degree fundus photography after pupillary dilation. An ophthalmologist or trainee ophthalmologist took the photographs using a Topcon TRCNW100 digital fundus camera (Topcon Corporation). The photos were centred on the fovea with a 45 degree field of view.

In line with many other papers reporting accuracy of screening photographs for DR, our paper has included data from both eyes to allow comparative assessment of accuracy of our study alongside others. ${ }^{10}$ The photos from individual patients were not assessed in pairs, but in a random order to minimise bias.

Various screening modalities for DR have been studied to meet the increased demand for screening, with variable rates of success. These include screening using mydriatic and nonmydriatic photography, and examination by different health professionals, including physicians, general practitioners and optometrists. To date, the ETDRS 7-field fundus photographs and ETDRS protocol are the only validated reference standard for detecting and staging of DR. Others have evaluated the validity of single-field fundus photography by comparing the accuracy of diagnosis against either a clinical gold standard of slit-lamp fundus examination ${ }^{13-16}$ or an imaging gold standard of 7 -field fundus photographs. ${ }^{17-19}$ Although dilated stereoscopic fundoscopy has an inherent weakness in that it cannot be validated or verified as there is no permanent record, it is by far the most commonly used method of DR evaluation in clinical practice. It allows clinicians to determine the presence of DR or VTDR. Thus, in our study, we set out to assess the validity of a screening method compared with standard clinical practice.

A single clinician (JL) graded the fundus photographs in a masked fashion using ETDRS criteria. Photos were graded as having either "any DR" or "VTDR" (ie, CSMO and/or PDR). The photo grades were compared with clinical grades from the dilated slitlamp fundus examination, which was used as the gold standard for comparison. A subset of the fundus photos were selected at random using a pseudorandom number generator, and regraded by the original grader in a masked fashion to establish the intragrader reliability for photo grading.

The statistical analysis was performed using SAS 9.1 (SAS Institute).
Test statistics, 95\% CI, kappa coefficients as a measure of intra-examiner assessment and $P$ values are presented.

\section{Results}

From the 1884 individuals recruited across 30 remote communities, 1040 had self-reported DM. Fundus photos were available for 396 of those patients (792 eyes). The remaining participants were not photographed because of space limitations of the light plane used to access the remote communities, the camera being needed at other locations, or intermittent maintenance of the camera. Of the available images, 86 photographs were ungradable because of media opacity or defocus, leaving 706 fundus photographs that were used for analysis. Of the 360 patients included for analysis, 131 were male $(36 \%)$ and 229 were female (64\%). The mean age of patients was 48 years (range, 20-83 years; SD, 13 years). This was similar to the whole sample of 1040 patients with diabetes, where the mean age was 50 years (range, 20-93; SD, 14 years; $t=1.90$; $P=0.06)$, and $66 \%$ of patients were female $\left(\chi^{2}=0.88 ; P=0.38\right)$.

Any DR was detected in 163 eyes (23\%) by clinical examination and in 162 eyes (23\%) using fundus photography (Box 2, A). Of those with any DR detected by clinical examination, 52 (32\%) had minimal NPDR, 63 (39\%) had mild NPDR, 38 (23\%) had moderate NPDR, one (1\%) had severe NPDR, nine (6\%) had PDR and 42 (26\%) had CSMO.

VTDR was detected in 51 eyes (7\%) on clinical examination and in 78 eyes (11\%) using photo grading. Seven eyes with VTDR diagnosed clinically were not detected on photo screening, and 34 eyes were incorrectly diagnosed by photo grading as having VTDR (Box 2, B), leaving 44 eyes with VTDR (6\%).

The sensitivity, specificity and kappa values for detecting any DR were $74 \%$ (95\% CI, 67\%-80\%), 92\% (95\%CI, $90 \%-94 \%)$ and 0.67 (95\% CI, 0.60 0.74; $P<0.0001$ ), respectively (Box 3). The sensitivity and specificity for detecting VTDR were $86 \%$ (95\% CI, 77\%-96\%), 95\% (95\% CI, 93\%-97\%) and 0.65 (95\% CI, 0.55-0.76; $P<0.0001$ ), respectively (Box 3 ).

The kappa values for intragrader reliability for detecting any DR and 
2 Accuracy of single-field 45-degree fundus photography in detecting any diabetic retinopathy (DR)* and vision-threatening diabetic retinopathy (VTDR) in 360 patients (706 eyes) using clinical slit-lamp fundoscopy as the gold standard A: Any DR

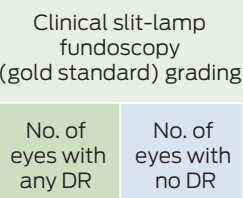
B: VTDR
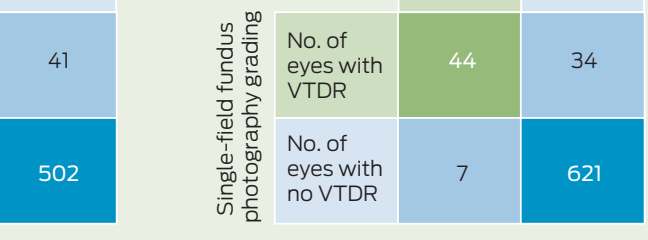

* Both of the categories "any DR" and "VTDR" include eyes with proliferative DR. large sample of Indigenous Australians using single-field dilated fundus photography compared with clinical examination. Our patient recruitment was somewhat limited by factors such as availability of instruments, and space and weight restrictions on light planes. However, this is unlikely to have introduced a systematic bias and we were able to obtain photographs of a representative sample of 706 eyes.

$\mathrm{CSMO}$ is the most common cause of VTDR. However, in the absence of stereoscopic views, as with single-field digital photos, assessment for VTDR is limited to detecting associated features, such as lipid exudates or haemorrhages, rather than retinal thickening. Our results show that the false-positive grades on fundus photographs were largely attributable to pigment hyperplasia or depigmentations that mimicked the appearance of lipid and haemorrhages. This problem can be overcome in the clinical setting by using stereo-photos and red-free filters, which were not available for the model of camera used in this study. However, a false-positive diagnosis of VTDR is preferable as it will not lead to vision loss compared with a false-negative diagnosis.

Cost analysis studies have demonstrated the potential cost-effectiveness of photo-screening in remote settings in maintaining both a high level of sight-years as well as being cheaper than visiting specialist programs. ${ }^{26,27}$

The NHMRC recommends that DR screening modalities need to be costeffective and easy to administer, and to have a sensitivity of at least $60 \%$ and specificity levels of $90 \%-95 \%{ }^{20}$ The accuracy of the photo-screening in our study exceeds this minimum requirement, with sensitivities of $74 \%$ and $86 \%$ for detecting any DR and VTDR, respectively. Likewise, the specificities for detecting any DR and VTDR meet specificity with mydriasis. 22 Other studies have shown that photos without mydriasis were often of poor quality and unable to be graded, especially in older patients and in the presence of media opacities. ${ }^{18,19}$ The higher failure rate of fundus photography in our study (11\%; 86/792) compared with the reported failure rate of dilated fundus photography $(4 \%-8 \%)^{14-16,18}$ may have resulted from a higher prevalence of media opacities, such as corneal scarring and cataract, among Indige-

Most of the studies of fundus photographs to date are based largely on white populations. ${ }^{14,16,18,19}$ Previous studies evaluating fundus photography as a screening tool in Indigenous Australians were based on non-mydriatic fundus photographs with smaller sample sizes. ${ }^{24,25}$ To our knowledge, this is the first study that assesses the accuracy of detecting DR and VTDR in a reliability for detecting any diabetic

3 Diagnostic characteristics and interrater reliability for detecting any diab
retinopathy (DR) and vision-threatening diabetic retinopathy (VTDR)

\section{Diagnostic characteristics}

Sensitivity

Specificity

Positive predictive value

Negative predictive value

Positive likelihood ratio

Negative likelihood ratio

Kappa

\begin{tabular}{cc} 
Any DR (95\% CI) & VTDR $(95 \% \mathrm{CI})$ \\
\hline $74 \%(67 \%-80 \%)$ & $86 \%(77 \%-96 \%)$ \\
$92 \%(90 \%-94 \%)$ & $95 \%(93 \%-97 \%)$ \\
$75 \%(68 \%-81 \%)$ & $56 \%(45 \%-67 \%)$ \\
$92 \%(90 \%-94 \%)$ & $99 \%(98 \%-100 \%)$ \\
$9.8(7.2-13.4)$ & $16.6(11.8-23.5)$ \\
$0.3(0.2-0.4)$ & $0.1(0.1-0.3)$ \\
$0.67(0.60-0.74)$ & $0.65(0.55-0.76)$
\end{tabular}


the NHMRC guideline of greater than $90 \%$, at $92 \%$ and $95 \%$, respectively. These values are comparable to the reported sensitivities and specificities of studies that compared diagnostic accuracy of single-field fundus photography with that of clinical examination. The sensitivities of detecting any DR have been reported from 38\% to $96 \%$, and specificities from $79 \%$ to $98 \% .^{13-16}$ Although definitions of VTDR vary in different studies, the sensitivities and specificities were higher for detecting VTDR, with ranges of $82 \%-100 \%$ and $70 \%-100 \%$, respectively. ${ }^{13-16}$ In addition, the measure of reliability of inter-examiner and intra-examiner assessment (kappa coefficient $\kappa)$ showed a moderate level of agreement $(\kappa=0.67$ and 0.65 for any DR and VTDR, respectively).

Our study highlights the strength and simplicity of single-field fundus photography for DR screening; however, there is still room for improvement. Future research could investigate the adjunctive use of optical coherence tomography (OCT) to improve screening for CSMO within this population. The development of conveniently portable OCT would reduce the risk of false-negative gradings, confirm tentative diagnoses of retinal thickening, and the images generated could enhance patient comprehension of what is happening in DR. In addition, while single-field fundus photography may be feasible for screening for DR in a remote setting, if this approach is to be considered, future research must also establish whether there is likely to be sufficient uptake of this modality by the workforce currently in place in remote communities. Thus, measures such as this should be undertaken after consultation with current health providers to ensure that the desired outcome is achieved.

\section{Conclusion}

Our results show that single-field dilated fundus photography in Indigenous Australians meets the minimum screening requirements recommended by the NHMRC. It also has adequate sensitivities, specificities and repeatability for detecting DR to determine need for referral or repeat screening frequencies. It is therefore a valid screening tool for DR in remote communities within Central Australia. This technique may be used to complement visiting specialist eye care services to this region with acceptable accuracy. Providing optometrists or other trained technicians who visit the communities with fundus cameras or installing fundus cameras in the medical clinics of remote communities and training local staff to recognise signs of DR may help identify patients who require referral in a timely manner. Alternatively, remote clinics can be linked via high-speed internet connection to reading centres and/or ophthalmologists who can give their opinion and make recommendations on further action.

Acknowledgements: We would like to thank Renu Raju, Susan Wearne, Kathryn Billing, Tim Gray, Ilesh Patel, Jwu Jin Khong, John Chang, Shane Durkin and Douglas Parker, without whom this project would not be possible. We would also like to thank Vanessa Davies and Sarah Ford for their support.

Competing interests: This study is supported by the Ophthalmic Research Institute of Australia and the NHMRC Centres for Clinical Research Excellence.

Received 8 Apr 2012, accepted 26 Oct 2012.

1 Intensive blood-glucose control with sulphonylureas or insulin compared with conventional treatment and risk of complications in patients with type 2 diabetes (UKPDS 33). UK Prospective Diabetes Study (UKPDS) Group. Lancet 1998; 352: 837-853.

2 Klein R, Klein BE, Moss SE, Cruickshanks KJ. The Wisconsin Epidemiologic Study of Diabetic Retinopathy: XVII. The 14-year incidence and progression of diabetic retinopathy and associated risk factors in type 1 diabetes. Ophthalmology 1998; 105: 1801-1815.

3 Resnikoff S, Pascolini D, Etya'ale D, et al. Global data on visual impairment in the year 2002. Bull World Health Organ 2004; 82: 844-851.

4 Tapp RJ, Shaw JE, Harper CA, et al; AusDiab Study Group. The Prevalence of and factors associated with diabetic retinopathy in the Australian population. Diabetes Care 2003; 26: 1731-1737.

5 Xie J, Arnold AL, Keeffe J, et al. Prevalence of selfreported diabetes and diabetic retinopathy in Indigenous Australians: the National Indigenous Eye Health Survey. Clin Experiment Ophthalmol 2011; 39: 487-493.

6 Landers J, Henderson T, Abhary S, Craig J. Prevalence and associations of diabetic retinopathy in Indigenous Australians within central Australia: the Central Australian Ocula Health Study. Clin Experiment Ophthalmol 2010; 38: 393-397.

7 Photocoagulation for diabetic macular edema. Early Treatment Diabetic Retinopathy Study report number 1. Early Treatment Diabetic Retinopathy Study research group. Arch Ophthalmol 1985; 103: 1796-1806.

8 Schoenfeld ER, Greene JM, Wu SY, Leske MC. Patterns of adherence to diabetes vision care guidelines: baseline findings from the Diabetic Retinopathy Awareness Program. Ophthalmology 2001; 108: 563-571.

9 Lee PP, Feldman ZW, Ostermann J, et al. Longitudinal rates of annual eye examinations of persons with diabetes and chronic eye diseases. Ophthalmology 2003; 110: 1952-1959.

10 Bragge P, Gruen RL, Chau M, et al. Screening for presence or absence of diabetic retinopathy: a meta-analysis. Arch Ophthalmol 2011;129: 435-444.

11 Landers J, Henderson T, Craig J. Central Australian Ocular Health Study: design and baseline description of participants. Clin Experiment Ophthalmol 2010; 38: 375-380.

12 Grading diabetic retinopathy from stereoscopic color fundus photographs--an extension of the modified Airlie House classification. ETDRS report number 10. Early Treatment Diabetic Retinopathy Study Research Group. Ophthalmology 1991; 98 (5 Suppl): 786-806.

13 Williams GA, Scott IU, Haller JA, et al. Single-field fundus photography for diabetic retinopathy screening: a report by the American Academy of Ophthalmology. Ophthalmology 2004; 11 : 1055-1062.

14 Joannou J, Kalk WJ, Mahomed I, et al. Screening for diabetic retinopathy in South Africa with 60 degrees retinal colour photography. J Intern Med 1996; 239: 43-47.

15 Maberley D, Cruess AF, Barile G, Slakter J. Digital photographic screening for diabetic retinopathy in the James Bay Cree. Ophthalmic Epidemiol 2002; 9: 169-178.

16 Herbert HM, Jordan K, Flanagan DW. Is screening with digital imaging using one retinal view adequate? Eye (Lond) 2003; 17: 497-500.

17 Taylor DJ, Fisher J, Jacob J, Tooke JE. The use of digital cameras in a mobile retinal screening environment. Diabet Med 1999; 16:680-686.

18 Pugh JA, Jacobson JM, Van Heuven WA, et al. Screening for diabetic retinopathy. The wideangle retinal camera. Diabetes Care 1993; 16: 889-895.

19 Lin DY, Blumenkranz MS, Brothers RJ, Grosvenor DM. The sensitivity and specificity of single-field nonmydriatic monochromatic digital fundus photography with remote image interpretation for diabetic retinopathy screening: a comparison with ophthalmoscopy and standardized mydriatic color photography. Am J Ophthalmol 2002; 134: 204-213.

20 National Health and Medical Research Council. Guidelines for the management of diabetic retinopathy. Canberra:Commonwealth of Australia, 2008. http://www.nhmrc.gov.au/ guidelines/publications/dil5 (accessed Jul 2012).

21 Hutchinson A, Mclntosh A, Peters J, et al. Effectiveness of screening and monitoring tests for diabetic retinopathy--a systematic review. Diabet Med 2000; 17: 495-506.

22 Lawrence MG. The accuracy of digital-video retinal imaging to screen for diabetic retinopathy: an analysis of two digital-video retinal imaging systems using standard stereoscopic seven-field photography and dilated clinical examination as reference standards. Trans Am Ophthalmol Soc 2004; 102: 321-340.

23 Landers J, Henderson T, Craig J. Prevalence and associations of cataract in Indigenous Australians within central Australia: the Central Australian Ocular Health Study. Clin Experiment Ophthalmol 2010; 38: 387-392.

24 Diamond JP, McKinnon M, Barry C, et al. Nonmydriatic fundus photography: a viable alternative to fundoscopy for identification of diabetic retinopathy in an Aboriginal population in rural Western Australia? Aust NZ J Ophthalmol 1998; 26: 109-115.

25 Harper CA, Livingston PM, Wood C, et al. Screening for diabetic retinopathy using a nonmydriatic retinal camera in rural Victoria. Aust NZ J Ophthalmol 1998; 26:117-121.

26 Lee SJ, McCarty CA, Taylor HR, Keeffe JE. Costs of mobile screening for diabetic retinopathy: a practical framework for rural populations. Aust J Rural Health 2001; 9: 186-192.

27 Maberley D, Walker H, Koushik A, Cruess A. Screening for diabetic retinopathy in James Bay, Ontario: a cost-effectiveness analysis. CMAJ 2003; 168: 160-164. 